\title{
Defined Criterion Group Option Relationship Comment
}

National Cancer Institute

\section{Source}

National Cancer Institute. Defined Criterion Group Option Relationship Comment. NCI

Thesaurus. Code C94178.

Additional description of the criterion group option relationship. 